\title{
Application of Tele-Ultrasound in Emergency Medical Services
}

Mei-Ju Su, Ph.D. (ABD), ${ }^{1}$ Huei-Ming Ma, M.D., Ph.D., ${ }^{2}$ Chow-In Ko, M.D., ${ }^{2}$ Wen-Chu Chiang, M.D., ${ }^{2}$ Chih-Wei Yang, M.D., ${ }^{2}$ Sao-Jie Chen, Ph.D., ${ }^{1}$ Robert Chen, B.S., ${ }^{3}$ and Heng-Shuen Chen, M.D., Ph.D. ${ }^{4}$

${ }^{1}$ Graduate Institute of Electronic Engineering, College of Electrical Engineering and Computer Science, National Taiwan University, Taipei, Taiwan.

2Department of Emergency Medicine, National Taiwan University Hospital, Taipei, Taiwan.

${ }^{3}$ Department of Human Biology, University of Toronto, Toronto, Canada.

${ }^{4}$ Department of Medical Informatics, National Taiwan University College of Medicine; Department of Family Medicine, National Taiwan University Hospital; and School of Medicine, National Taiwan University College of Medicine, Taipei, Taiwan.

\section{Abstract}

In emergency medical services, portable ultrasound scanners have the potential to become new-age stethoscopes for emergency physicians. For trauma cases in particular, portable ultrasound scanners can scan the chest and abdomen of emergency patients both rapidly and conveniently. This study describes the development of tele-ultrasound for pre-diagnosis in a medical emergency setting as a part of the updated Mobile Hospital Emergency Medical System (MHEMS). An emergency medical technician can provide an emergency physician with a patient's ultrasound images and medical information during the patient's pre-hospitalization and transportation period using a combination of the MHEMS, the portable ultrasound scanner, and the onboard $3 G$ communication capabilities. The MHEMS includes a Dispatch and Mission Control Center that facilitates the communication between the Emergency Department of a specified hospital, the systems aboard the ambulance. Early receipt of information relevant to the patient will enhance pre-diagnosis options for on-duty emergency physicians and allow for a hospital's emergency depart ment to promptly prepare necessary surgical instruments or beds. Furthermore, emergency medical technicians can also obtain instructions from on-duty physicians to enhance damage and disaster control ability in critical moments.

Key words: emergency medical services; tele-ultrasound; Mobile Hospital Emergency Medical System; blunt abdominal trauma

\section{Introduction}

$\mathrm{n}$ general, emergency medical service is composed of pre-hospitalization and in-hospital periods. The most important tasks in the pre-hospitalization period are preliminary identification of the victims/patients, activation of emergency medical services, cardiopulmonary resuscitation and early defibrillation in the case of patients suffering from cardiac arrest, early advanced life support, first-aid treatment on the scene, and transportation of the patient(s). ${ }^{1}$ In the 1970s, Americans developed the first substantial medical emergency network in order to provide emergency care for the wounded during the Vietnam War. Emergency medical technician (EMT) training courses were first held at that time. In the 1980s, trauma centers and protocols were established and mortality rates rapidly declined due to the shortening of pre-hospitalization times. In emergency medicine, the "Golden Hour" is considered to be the first 60 minutes after the occurrence of multisystem trauma. It is widely believed that the victim's chances of survival are greatest if they receive definitive care in the operating room within this Golden Hour. ${ }^{2}$ Thus, any system that can shorten the pre-hospitalization and/or transportation period can have a significant impact on patient survivability.

However, the identification of intra-abdominal injuries in the case of patients with blunt trauma can be difficult, although essential. At present, the assessment of blunt abdominal trauma (BAT) at an emergency department presents a diagnostic problem. Clinical examination of the abdomen can be unreliable, especially in severely injured or unconscious patients. Investigative techniques most commonly employed include diagnostic peritoneal lavage (DPL) and computed tomography 


\section{TELE-ULTRASOUND IN EMERGENCY MEDICAL SERVICES}

(CT) scanning, both of which have disadvantages. For example, DPL carries a small but significant risk of intra-abdominal injury and is time consuming. ${ }^{3}$ CT scans require specialized personnel and equipment that may not be immediately available to critical patients with BAT. ${ }^{4}$ Focused Assessment with Sonography for Trauma (FAST) is a technique initially developed by Rozycki et al. (1993) for the ultrasound assessment of abdominal trauma. ${ }^{5}$ North American studies have shown it to be an effective and sensitive technique for the detection of BAT and equally accurate in the hands of nonradiologists and radiologists alike. ${ }^{6}$ There is further evidence from American and European studies that focused, abdominal emergency ultrasonography can reliably detect the presence of free intraperitoneal fluid. In the context of trauma, this is often taken to be a diagnostic sign of internal hemorrhaging. ${ }^{7}$ FAST utilizes a limited four-view abdominal scan, which is a rapid procedure that can be performed during resuscitation to detect the presence of internal injury. As smaller and more-portable units were being developed, some people started to feel that ultrasound might be the future stethoscope of emergency medicine. ${ }^{8}$

In Taiwan, on islands and remote districts, which may be deficient in medical resources such as radiology capabilities and which require longer transportation times, FAST could greatly improve emergency medical services by identifying important and priority injuries, if EMTs could be trained to collect the data appropriately. Taiwan began to provide EMT training in 1995. Taiwan's EMTs must at minimum pass primary first-aid training and obtain certification to conduct rescues. They differ from an "early rescuer" in that they are also responsible for transporting patients to a hospital. Taiwanese EMTs are classified as either EMT-I, EMT-II, or EMT-P. EMT-Is have completed 60 hours of professional training, EMT-IIs have completed 264 hours of professional training, and EMT-Ps have completed 1,280 hours of professional training. The duties of EMT-Is and EMTIIs include basic life support, while an EMT-P's task is advanced life support. The National Taiwan University Hospital (NTUH) is the first medical center in Taiwan to collaborate with the National Fire Agency comprehensive support for the training of EMT-Ps. ${ }^{9}$

EMTs also have the responsibility of providing life support and acute trauma management. However, even EMT-Ps do not commonly possess the skill and knowledge to perform and to interpret the results of more advanced examinations such as FAST in the pre-hospitalization period. Early diagnoses using these examinations may be critical to providing valuable information to on-duty physicians and can enhance preparations for emergency interventions such as surgery. If the emergency physician can remotely instruct EMTs to perform and obtain imagery from systems such as FAST via an online command system, pre-diagnosis can be significantly improved in terms of time and accuracy. The purpose of this study was to develop a system comprising a tele-ultrasound device integrated with a mobile healthcare information system for EMTs to use on an ambulance and/ or at an emergency response site. The resultant imaging and relevant medical information could then be transmitted via a broadband wireless network to emergency physicians at a hospital to enhance the pre-diagnosis and decision support in emergency medical services.

\section{Methods}

In developing a tele-ultrasound application in emergency medical service, the following steps were taken. First, an updated version of the Mobile Hospital Emergency Medical System (MHEMS) was created, with expanded function models on all three subsystems. Second, the mobile FAST examination procedure was standardized by experts in emergency medicine. Third, after analyses of user requirements, two user interfaces using tele-ultrasound applications were designed to be used by EMTs and emergency physicians. Last, test trials and case simulations on patients in emergency settings were conducted.

\section{SYSTEM ARCHITECTURE OF NEW MHEMS}

The information system used in the study, integrating an updated MHEMS and a wireless hospital system using WiFi and VoIP technology at NTUH (Fig. 1), aims to provide emergency physicians with a pre-diagnostic aid through the application of a portable ultrasound system on ambulances. ${ }^{10,11}$ The three subsystems of MHEMS are located at Dispatch and Mission Control Center, on board ambulances, and in the emergency departments of selected hospitals. The mobile Internet communication between the three subsystems is based on a $3 \mathrm{G}$ communication protocol added to the existing General Packet Radio Service (GPRS), which was a 2.5G system. The 3G network is based on a Universal Mobile Telecommunications System (UMTS). An upgraded UMTS system or high-speed downlink packet access (HSDPA), sometimes called 3.5G, supports higher downlinks up to 3.6 megabytes per second and the uplink rates of 384 kilobits per second. HSDPA possesses powerful capabilities in transferring voice, video, and data information even while being used on a mobile platform such as an ambulance. In our system, the transferred data includes ultrasonography video, ultrasonography images, vital signs, and other medical information relevant to the patient.

\section{SUBSYSTEM AT THE DISPATCH AND MISSION CONTROL CENTER}

This subsystem plays the role of the major data-processing and application center for emergency medical services. The MHEMS server at the Control Center includes Decision Support System and Geographic 
SU ET AL.

Information System (GIS) capabilities, and is designed to connect to the Internet and cooperate with participating fire-fighting departments.

\section{SUBSYSTEM AT AMBULANCE}

The main components of the ambulance system of an updated MHEMS include a Direct Medical System (DMS) InnovaSound (Direct Medical Systems, Pleasanton, CA) 3.5-MHz ultrasound probe (Fig. 2), as well as various vital sign sensors for temperature, heart rate, oxygen saturation, blood pressure, electrocardiogram (ECG), and respiration rate. The updated system also possesses a Global Positioning System (GPS) receiver, various GPRS/UMTS/HSDPA modules, an ultramobile personal computer, a Personal Digital Assistant (PDA), and three digital cameras separately installed on the PDA, on the ultra-mobile personal computer (UMPC), and inside the ambulance. ${ }^{12}$ The UMPC is the workstation that integrates the information collected by the ultrasound probe, the vital signs sensors, and the camera. It then transfers the collected data to a hospital's emergency department via a HSDPA wireless telecommunication network. The InnovaSound $3.5 \mathrm{MHz}$ is a general purpose portable ultrasound system with U.S. Food and Drug Administration clearance to be marketed in April 2007. This portable ultrasound system has an ultrasonic probe connecting to a UMPC via USB, and can mass less than a kilogram (Fig. 2).

\section{SUBSYSTEM AT EMERGENCY DEPARTMENT OF HOSPITAL}

This subsystem integrates into the Emergency Information System (EIS) of the NTUH to receive information from incoming ambulances

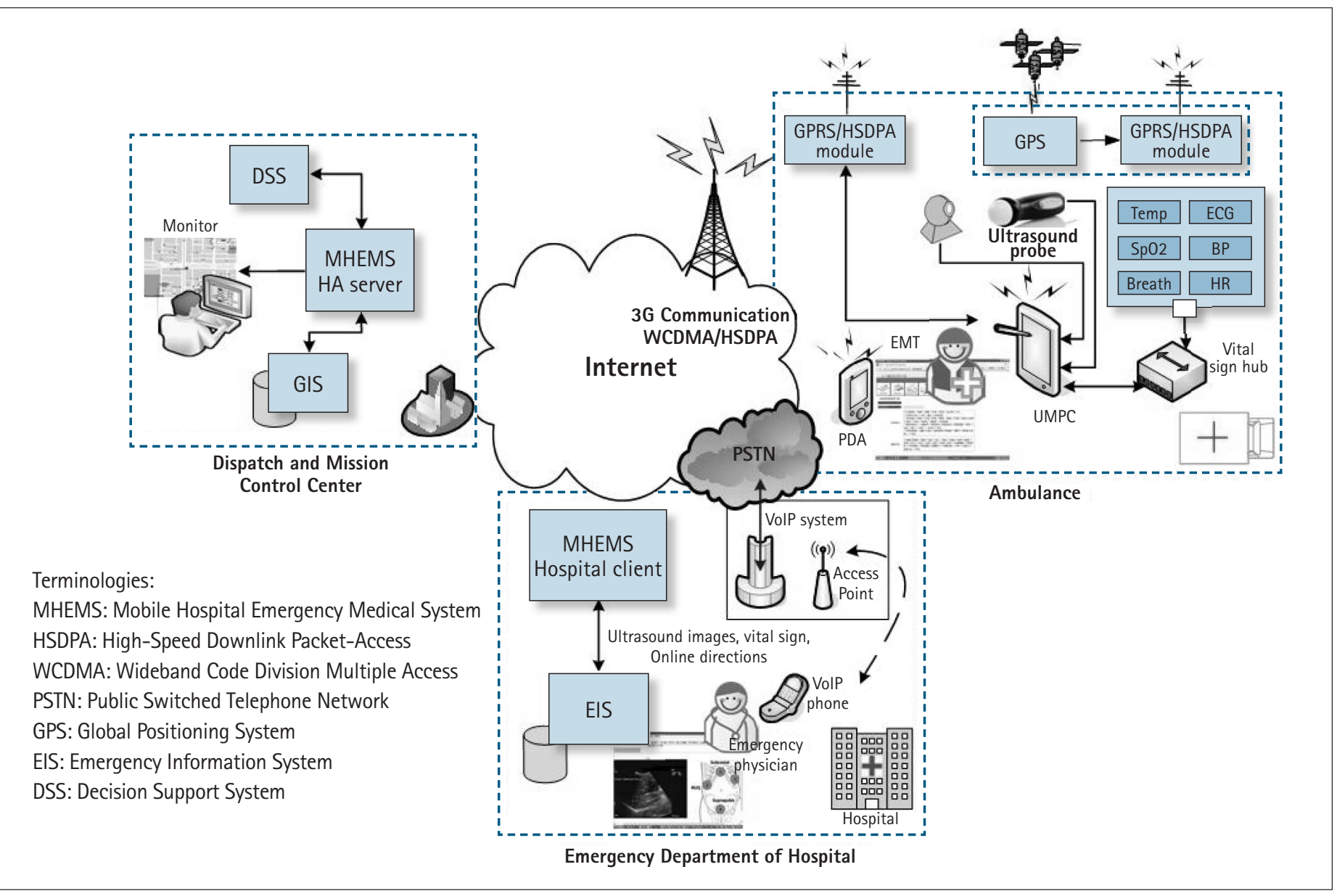

Fig. 1. System architecture. 
via the Internet. Furthermore, this subsystem is also linked to the wireless hospital system and can provide mobile communication between EMTs, emergency physicians, and other related personnel through WiFi and VoIP services. The wireless hospital system enables seamless and spaceless collaboration among medical professionals and administrative staff to provide continuous and comprehensive healthcare for patients inside and outside of the hospital. This collaboration can be especially important in emergency medical situations, where time is critical in preparing for surgical interventions or admissions to ICUs and other inpatient wards.

\section{STANDARD PROCEDURE OF MOBILE FAST EXAMINATION}

The tele-ultrasound module based on a USB-compatible ultrasound probe connected to a UMPC is the key component of a mobile FAST examination. In order to standardize the mobile FAST examination procedure, experts in the field of emergency medicine and medical informatics worked together to formulate the optimal ultrasound examination to be performed by EMTs on ambulances and at emer-

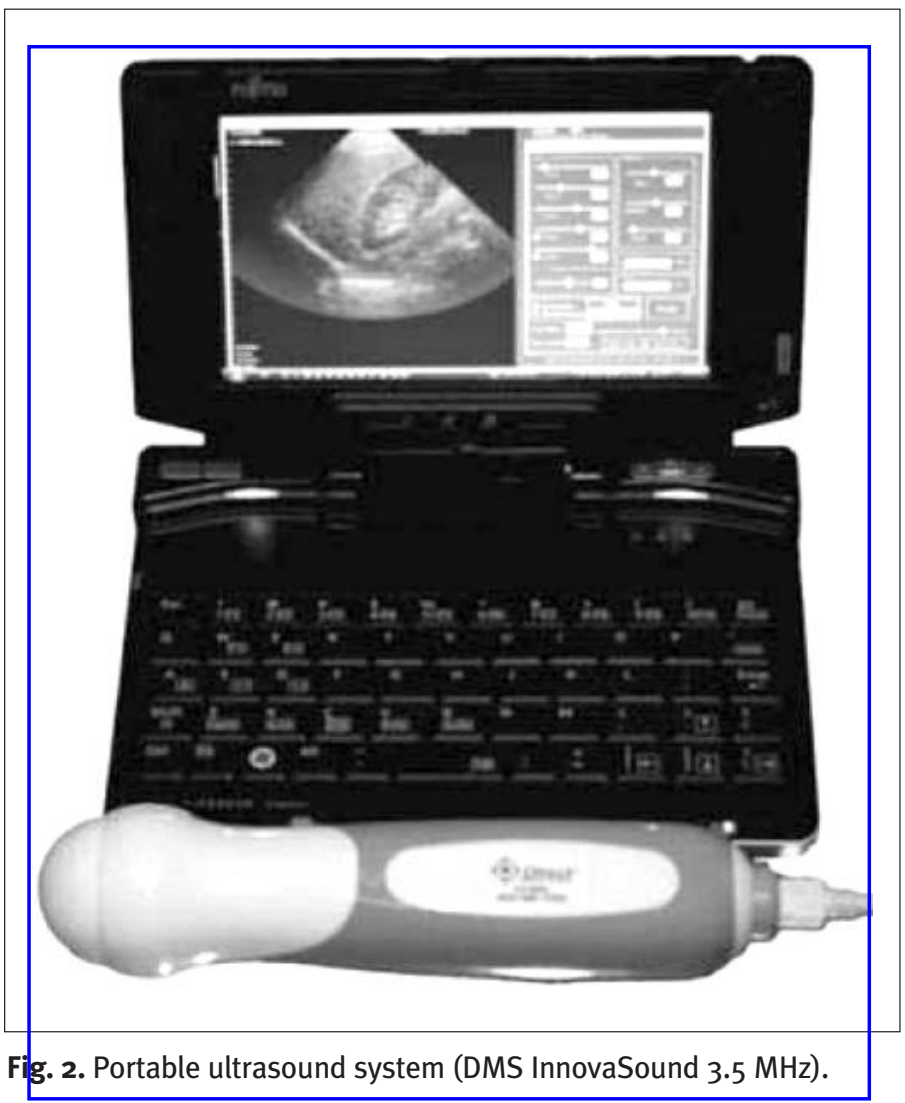

gency response sites. The examination procedure will be integrated into a pre-hospital workflow for emergency medical service training. FAST examination focused on four views:

- Subcostal view: Images obtained from the subxiphoid region of the chest with the ultrasound beam projecting into the coronal plane or directing the beam retrosternally. This view will focus on the heart and pericardial fluid.

- Right upper quadrant view: Images obtained from the right midaxillary line between the 11th and 12th ribs with the beam in a craniocaudal plane to examine the liver, kidney, diaphragm, and possibly fluid in Morrison's pouch (which represents space between the capsule of the liver and the fascia).

- Left upper quadrant view: Images obtained from the left midaxillary line between the 10th and 11th ribs with the ultrasound beam in a craniocaudal plane to examine the spleen, kidney, diaphragm, as well as the pleural, peritoneal, and retroperitoneal fluid.

- Suprapubic view: from the abdominal midline, approximately 4 $\mathrm{cm}$ superior to the symphysis pubis, with the beam projecting transversely and angled downward into the pelvis to examine the bladder. The probe is then rotated $90^{\circ}$ to move the beam onto the sagittal plane, thus providing a view of the bladder, rectum, and rectovesical pouch. EMTs performed the mobile FAST examination using the DMS InnovaSound software installed on the UMPC. After identifying the patient to the system, ultrasound scans were then performed on the four views, with each lasting for at least 5 seconds. All scans were recorded in video form. At the end of each scan, and after locating the most accurate position for the probe, a single still image is also taken. The suprapubic view consisted of two scans including those performed from both the transverse and sagittal planes. A standard four-view examination can be completed in 2 minutes.

\section{MULTIDISCIPLINARY TEAMWORK}

This study used a multidisciplinary approach that integrated the researcher team, research meetings, system deployment program, and mobile FAST examination training course with the Taiwanese College of Medicine, College of Electric Engineering and Computer Science, and the National Taiwan University Hospital.

- Research team: consisted of attending and resident physicians of the emergency department at the NTUH, as well as specialists in Medical Informatics, Electronics Engineering, and the Hospital Information Service Department.

- Research meetings: regular meetings were held to facilitate discussion among the users and system developers. User require- 
ments analyses were done thoroughly to determine the system function specifications.

- System prototyping was developed after the creation of the first draft of specification. The tele-ultrasound system consisted of a package of updated functions added to the previous MHEMS. The updated system was deployed and distributed to three separate locations according to subsystems. The server intended to be located at the mission control and dispatch center function was instead installed at the Department of Medical Informatics at the College of Medicine, National Taiwan University. The user/ clients components were installed on a desktop PC located in the hospital's emergency room and on the tele-ultrasound UMPC located onboard an ambulance.

- Training Course of mobile FAST: A 4-hour mobile FAST course was designed and conducted for EMTs by both an attending physician of emergency medicine and an expert in medical informatics. The course will be applied to EMT-Ps first and gradually extend to lower level EMTs. The outline of the course is the following:

- Introduction to the tele-ultrasound system.

- The portable ultrasound unit: USB ultrasound probe and UMPC.

- Standardized mobile FAST procedure.

- Practice of FAST examination.

- FAST competency test (15 minutes).

\section{Results}

\section{USER REQUIREMENT ANALYSIS}

User requirement analyses were done by interviewing both emergency physicians and EMTs at the NTUH. Several Joint Requirements Development Meetings were held among specialists in emergency medicine, medical informatics, and electronic engineering. A prototype system was then developed after the creation of the first software requirement specification draft. The main functions of the tele-ultrasound system are follows:

- Electronic emergency medical records: including basic data, vital signs, important history, causes and complaints, management, and FAST 4-views. An abdominal figure with marked regions of all four views is provided, as well as an interfacing supporting the convenient selection and uploading of still and video ultrasound imagery to the MHEMS server.

- Electronic Map: incorporated with GIS and GPS functions. The Electronic Map will show the respective positions of the ambulance and hospital.

- Dispatch and Mission Control: including a user interface opti- mized for the functions of the dispatching and tracking of EMTs and ambulances. Near-instantaneous updated status reports can be viewed from this module.

\section{TELE-ULTRASOUND WORKFLOW IN EMS}

After determining the user requirements, detailed workflows for the ambulance, the emergency room, and the dispatch and mission control center were defined.

\section{EMTS ON AN AMBULANCE}

- Obtain case information of incident report and duty records of the ambulatory service from a server based at the Dispatch and Mission control center.

- Provide FAST 4-views (right flank, left flank, subcostal, and pelvic ultrasound scan), vital signs measurement. Also fill in forms for vital signs, complaints, histories and management, as well as basic information of the patient using the emergency system interface.

- Allow EMTs to cooperate with emergency physicians to obtain consults in a timely fashion.

- Transmit the patient's data to a workstation at the Dispatch and Mission control center.

\section{PHYSICIAN AT EMERGENCY ROOM}

- Obtain the entire duty record of the hospital from the Dispatch and Mission control center. Also trigger a flashing signal at the emergency room before the arrival of a new patient.

- Obtain FAST 4-views in the form of ultrasonography videos or images taken by EMTs to provide emergency physicians with pre-diagnoses and early preparation.

- Query all of the patient's data from the ambulance through the emergency system interface.

- Allow emergency physicians to support EMTs through the VoIP system and updated MHEMS.

- Obtain an Estimated Time of Arrival (ETA) from ambulances.

- Store the choice of emergency treatment to Emergency Information System (EIS).

\section{DISPATCH AND MISSION CONTROL CENTER}

- Define the district of each fire brigade and hospital.

- Accept case reports and input locations of accidents. Also retrieve or query for the next-of-kin's phone number and other relevant data.

- Record the duty logs of the ambulance, such as arrival and departure times to accident sites and the hospital. 
- Calculate the ETA of ambulances before they arrive at the hospital.

- Monitor the progress of the ambulances.

- Print a duty report.

\section{PROTOTYPING}

A prototype was developed according to the user requirements and workflows. EMTs and emergency physicians may log in to use the Web-based client. The system would display different input and user interface options depending on the type of user (Figs. 3 and 4). The tele-ultrasound system was installed on a UMPC with touch-screen capabilities, and a USB console that could link different biosensors for monitoring important vital signs and other physiological parameters. The hardware, software, development tools, and their distribution were the following:

\section{HARDWARE}

Dispatch and mission control center. (1) MHEMS server station: Window Server 2003 as the web server and database server

Emergency room. (1) EIS workstation: a Desktop PC for emergency physician at emergency room linked to both the EIS of the hospital and to the MHEMS server as a client.

EMT. (1) Portable Ultrasound workstation: a UMPC for EMTs equipped with a Portable Ultrasound Probe (DMS Innova Sound USB interface with the imaging software DMS InterSonoScan running on the UMPC) (Fig. 2). (2) Communication module: 3.5 G- and digital camera-enabled, capable of accessing the Internet and of videoconferencing. (3) Bio-Sensor modules: a USB console that can connect sensor modules including heart rate, blood pressure, temperature, ECG, respiration rate, and $\mathrm{SpO}_{2}$ meters.

Ambulance. (1) Localization module: GPS/GPRS module installed at the ambulance for locational and positional reports to mission control center.

Software and developmental tools. (1) NET Framework; (2) Apache Framework; (3) PHP; (4) MySQL; (4) Java Runtime Engine.

User interface. (1) Ambulance client. EMTs can input relevant medical information such as a patient's vital signs, cause of emergency and chief complaints, important history, and management. For critical examinations such as FAST directed at abdomen, a function module with a visual interface could be initiated after completing all four views (right flank, left flank, subcostal, and pelvis). By simply touching several buttons, all recorded video and still imagery can be transmitted via the 3.5G network and uploaded to the NHEMS server. (2) Hospital client. On the other hand, physicians of a hospital's emergency department can review the patient's medical informa- tion as well as the ultrasound imagery when they worked on duty at the hospital. The FAST 4-views presentation was designed for easy recognition and identifying critical internal injuries that emergency physicians can point to either of the 4-views representations of a patient's figure and discuss via voice or video communication with the EMT on the ambulance.

Case simulation. We also simulated using the tele-ultrasound system on a real case to evaluate the effectiveness of the system. A 21-year-

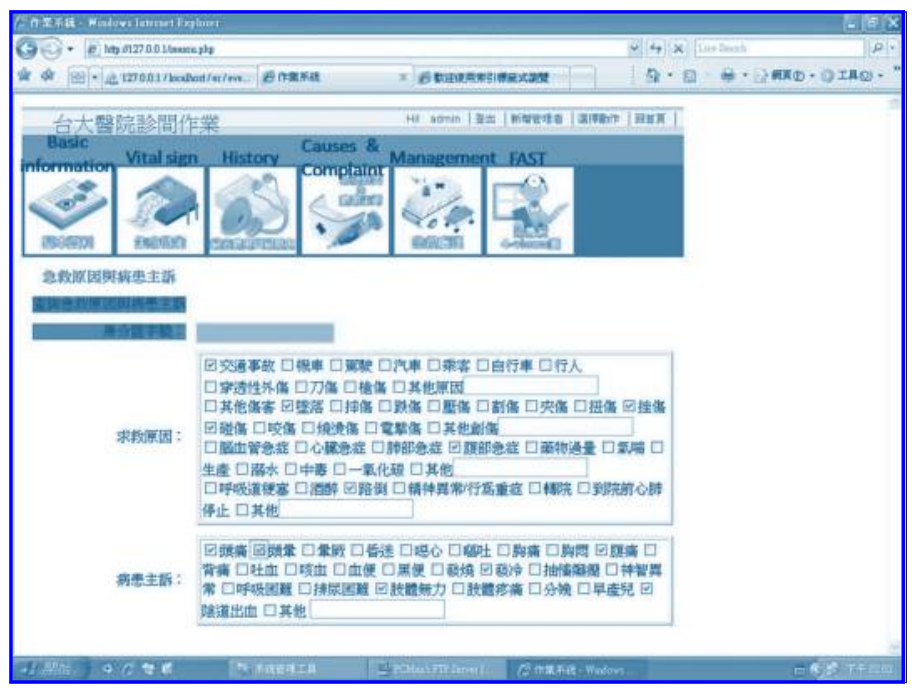

Fig. 3. Causes and Complaints interface.

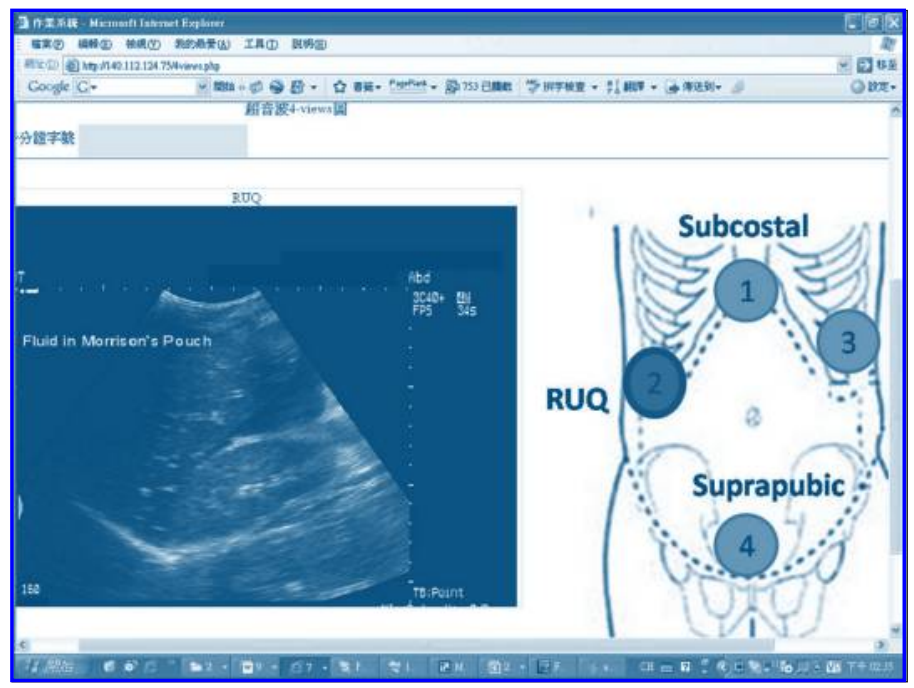

Fig. 4. FAST 4-views interface. 
old woman suffered a traffic accident at 12:53 P.M. The responding EMTs could not identify any internal hemorrhaging from her vital signs of: Glasgow Coma Scale (GCS): E4V5M6 (E, Eye opening; V, Verbal response; $\mathrm{M}$, Motor response), respiration rate: 16 , pulse rate: 73, blood pressure: $130 / 68$, body temperature: $35.5^{\circ} \mathrm{C}, \mathrm{SpO}_{2}: 100$, and skin color: normal. However, a FAST scan revealed free fluid in the patient's abdomen. Accordingly, when she arrived at the hospital, she was sent immediately to a surgery room at 13:30 P.M. to correct the suspected hemorrhaging.

What happened in the original case was that a CT scan revealed a hepatic hemorrhage and a laceration on the patient's right side. Due to the delayed discovery of these complications, the patient only entered a surgical room at 16:36 P.M. In the simulated case, the emergency physicians had already received information indicating a hepatic hemorrhage and would have time to make arrangements for surgery during the pre-hospitalization period. The time between the arrival of the patient at the hospital and the time it took for her to enter surgery was shortened so that the time of surgery in the simulated case was 3 hours earlier than the original case. An earlier attempt at surgical intervention would have greatly improved the survival rate of the patient. Therefore, the use of our emergency system, including the application of the tele-ultrasound technology, would have greatly improved the survival rates in such cases.

\section{Discussion}

\section{A RESTRICTED TOOL OF MEDICAL PROFESSIONALS}

One of the most important advances of medicine in recent years has been in the field of medical instrumentation technology, which has resulted in progressively enhanced efficiency in diagnoses and effective treatments. On the other hand, the emergence of broadband, mobile, and wireless network applications in information, communication, and technology (ICT) industries has greatly shortened the distance between people in terms of rapid and real-time communication systems. The resulting new era of telemedicine and e-health services has taken advantage of both technological advances and has successfully broken geographic and socioeconomic barriers. ${ }^{13}$ Two centuries ago, in the early 1800s, the stethoscope was first introduced to the field of medicine. One renowned physician, Sir John Forbis, remarked: "That it will ever come into general use, not withstanding its value...is extremely doubtful; because its beneficial application requires much time and gives a good bit of trouble both to the patient and the practitioner; because its hue and character are foreign, and opposed to all our habits and associations. There is even something ludicrous in the picture...of physicians using this device. ${ }^{14}$ However, time has proven that the stethoscope is one of the most important inventions in medicine, as it has given physicians the ability to examine patients through auscultation (aural) rather than merely through traditional methods of inspection (visual) and palpation (tactile). This expanded capability enhanced the physicians' ability to make accurate diagnoses for diseases of the internal organ diseases such as those in the lungs and the heart. Half a century ago, when ultrasound was first introduced to medicine, physicians were once again given a new set of capabilities; namely, they could now obtain noninvasive internal images of their patients. This allowed features of the internal organs to be considered when performing diagnoses, which was a powerful tool for physicians of that time. However, only the radiologist or those belonging to certain subspecialty fields were certified to use this new tool. There were hardly any standardized procedures that were performed by primary physicians and paramedical personnel.

\section{IS ULTRASOUND A FUTURE STETHOSCOPE?}

As the advance of ultrasound instrument technology progressed and the cost of such devices decreased, more and more general practitioners began to purchase and apply ultrasound techniques as an assistive diagnostic tool in daily practices. Office and bedside ultrasound screening began to be regularly performed in some clinics or community hospitals. In addition, more and more studies began to praise the effectiveness of ultrasound in clinical settings. Screening tests employing ultrasound have become more or less commonplace now, with scans requiring only a few minutes being employed in a variety of different medical fields such as the diagnosis of left ventricle dysfunctions in cardiology, ${ }^{15}$ prenatal, high-risk pregnancies in obstetrics, and internal hemorrhaging in abdominal trauma.

However, in the pre-hospital period of emergency medical situations, there are still few advances in the diagnostic tools available to EMTs other than the standard vital sign measurements. Since it is not realistic to field a medical professional on each and every ambulance, these EMTs are the only personnel on the ambulance that have received any meaningful medical training. Thanks to new developments in the information technology capabilities of UMPCs, both ultrasound probes and their associated computers can be as lightweight as $<1 \mathrm{~kg}$. The portability and instant imagery transmission abilities afforded by wireless broadband networks can allow EMTs to perform FAST tests within minutes upon arrival at the scene of the emergency. This timeframe allows for the pre-diagnosis and preparation for critical management to be accomplished within the "Golden Hour" in most cases. For all these improvement in quality of medical care, isn't the portable ultrasound system just like a future stethoscope? 


\section{TIME-CRITICAL PRESERVATION OF LIFE}

This study aims to add tele-ultrasound techniques and emergency physician support to improve the quality of pre-hospital medical emergency services. This system will enhance the speed of primary trauma assessment during the pre-hospital period, reducing the number of necessary CT scans and cutting medical costs. ${ }^{13}$ Once in the hospital, it will no longer be necessary to move patients to specialized sonography rooms for further sonographic evaluations. Accordingly, the service quality of the medical emergency service should be improved. In comparison, emergency physicians currently can only assess and diagnose patients after their arrival at the hospital. Furthermore, during the prehospitalization period, EMTs would be hard pressed to obtain sufficient instructions from emergency physicians via telephone to perform any critical assessments. Likewise, information flow from the EMTs to emergency physicians is equally impaired, thus hindering early assessments and diagnoses. In this study, the integration of an updated MHEMS system with a tele-ultrasound system not only improved communications between the EMTs on an ambulance, the emergency physicians at a hospital, and the dispatch and mission control center, but also provided all three parties with relevant medial information and necessary imagery for critical assessment or pre-diagnoses in a timely fashion. In this way, the technology empowered the proper personnel with extended capabilities and service quality.

\section{LIMITATIONS}

In Taiwan, where three quarters of the land mass is composed of mountainous terrain and isolated islets, medical resources are poorly distributed across the country. Thus, it would take a longer time to access a suitably equipped hospital or relevant medical specialists in emergency situations. On the other hand, EMTs en route to hospitals have plenty of time to conduct initial assessments if given the proper training. However, the poor communications infrastructure in nondeveloped areas would prevent EMTs from sending any initial assessments ahead to the hospital in any meaningful timeframe. For example, although GPRS is nearly fully distributed throughout the road system in Taiwan, 3G networks are not. To overcome this deficiency in high-speed transmission protocols, FAST test still images composed of the four previously discussed views could be stored and forward transmitted to reduce the data size and to surmount the problem of network interruptions. Other alternative communication network measures such as HAM radios and satellite communications also should be considered as backup solutions.

Although a diagnostically useful FAST scan is dependent on the skill level of the EMTs using the portable ultrasound unit, providing adequate tools is inarguably a prerequisite to adequate training. The competency of the onboard EMTs in performing initial assessment techniques such as FAST scans can be assured through comprehensive training, strictly defined workflow, and standardized practice guidelines.

\section{FUTURE PROSPECTS}

In the future, an improved system interface and standardized training protocols in the Taiwanese EMT-P training program should overcome any deficit in EMT skill level while using the portable ultrasound scanner. The usefulness of a portable ultrasound system in emergency medical service is not necessarily limited to trauma assessment. For example, in high-risk pregnancy or complicated labor and delivery, a portable ultrasound system could also be a very important tool for an EMT. It would be a worthwhile project to integrate obstetric ultrasound imagery techniques into a further-expanded MHEMS system. Adding to the portability of a tele-ultrasound system, a wearable computer sewn into clothes, complete with a head-mounted display and freehand data input, could become standard equipment for the next generation of EMTs.

\section{Disclosure Statement}

No competing financial interests exist.

\section{REFERENCES}

1. Cummins RO, Ornato JP, Thies WH, Pepe PE. Improving survival from sudden cardiac arrest: The "chain of survival" concept. A statement for health professionals from the Advanced Cardiac Life Support Subcommittee and the Emergency Cardiac Care Committee, American Heart Association. Circulation 1991;83:1832-1847.

2. Lerner EB, Moscati RM. The golden hour: Scientific fact or medical "urban legend"? Acad Emerg Med 2001;8:758-760.

3. Brooks A, Davies B, Smethhurst M, Connolly J. Prospective evaluation of nonradiologist performed emergency abdominal ultrasound for haemoperitoneum. Emerg Med J 2004;21:e5.

4. Rhea JT, Garza DH, Novelline RA. Controversies in emergency radiology: CT versus ultrasound in the evaluation of blunt abdominal trauma. Emerg Radio/ 2004;10:289-295.

5. Rozycki GS, Ochsner MG, Jaffin JH, Champion HR. Prospective evaluation of surgeons' use of ultrasound in the evaluation of trauma patients. J Trauma 1993;34:516-526.

6. Rozycki GS, Shackford SR. Ultrasound, what every trauma surgeon should know. J Trauma 1996;40:1-4.

7. Hilty WE, Wolfe RE, Moore EE. Sensitivity and specificity of ultrasound in the detection of intraperitoneal fluid. Ann Emerg Med 1993;22:921.

8. Ferris EJ. Ultrasound and emergency medicine. Emerg Radiol 2000;7:63-64.

9. Bullard MJ, Liaw SJ, Chen JC. Emergency medicine development in Taiwan. Ann Emerg Med 1996;28:542-548. 


\section{SU ET AL.}

10. Su MJ, Chen HS, Lin GS, Shih CL, Shyua FM, Su S, Cheng PH, Lin JW, Chen SJ. Application of wireless network in a medical emergency service network. Proceedings of Healthcom, 2005:23-25.

11. Chen HS, Cheng PH, Luh JJ, Chen SJ, Chen CL, Lai FP. Mobile health information system integrated with VolP technology in a wireless hospital. Proceedings of Healthcom, 2006:19-23.

12. Blaivas $\mathrm{M}$, Lyon $\mathrm{M}$, Duggal $\mathrm{S}$. Ultrasound image transmission via camera phones for over-reading. Am J Emerg Med 2005;23:433-438.

Address reprint requests to: Heng-Shuen Chen, M.D., Ph.D. Department of Medical Informatics National Taiwan University College of Medicine No. 1, Sec. 1, Ren-ai Road Taipei, 10051 Taiwan

13. Eysenbach G. What is e-health? J Med Internet Res 2001;32:e20.

14. TEC Jr.Will the stethoscope ever come into general use in clinical medicine? A strongly negative view expressed in 1821. Pediatrics 1978;62:113.

15. Alexander JH, Patel MR. Screening for structural heart disease: Time to stop listening and start looking. Am Heart J 2003;146:570-571.

Received: May 28, 2008

Accepted: May 28, 2008 